Internat. J. Math. \& Math. Sci.

Vol. 4 No. 1 (1981) 1-37

\title{
MOMENTS OF PROBABILITY MEASURES ON A GROUP
}

\author{
HERBERT HEYER \\ Mathematisches Institut der Universität \\ Auf der Morgenstelle 10 \\ D-7400 Tübingen, W. Germany \\ (Received October 28,1980 )
}

ABSTRACT. New developments and results in the theory of expectations and variances for random variables with range in a topological group are presented in the following order: (1) Introduction (2) Basic notions (3) The three series theorem in Banach spaces (4) Moment Conditions (5) Expectations and variances (6) A general three series theorem (7) The special cases of finite groups and Lie groups (8) The strong laws of large numbers on a lie group (9) Further studies on moments of probability measures.

KEY WORDS AND PHRASES. Probability Measures, Lie groups, ExpectaEions, Variances, Semigroup Homomorphism.

1980 MATHEMATICS SUBJECT CLASSIFICATION CODES: 60B15, 60J15 


\section{INTRODUCTION}

Moments of probability measures such as expectation and variance are important tools throughout probability theory and its applications. Moment conditions appear as helpful and crucial in almost all problems connected with the convergence in various senses of sequences of independent random variables. We shall restrict our attention to the almost sure convergence and the scope of the strong law of large numbers.

The aim of this paper is to report on new developments and results in the theory of expectations and variances for random variables taking their values in a topological group. The groups under discussion will be either the additive groups of topological vector spaces or locally compact groups. While the framework of a topological vector space admits a very natural extension of the notion of moments, some new ideas are needed in the case of an arbitrary locally compact group. Our exposition will be twofold: it will indicate the imitation procedures and at the same time describe the innovation available.

We start with the classical set up. Let $X$ be a real random variable on a probability space $(\Omega, O r, P)$. Its expectation and variance are defined by

$$
\begin{aligned}
& E(X):=\int X d P=\int X d P_{X} \text { and } \\
& V(X):=\int_{\Omega}(X-E(X))^{2} d P=\int_{\mathbb{R}}(X-E(X))^{2} P_{X}(d X)
\end{aligned}
$$


resp.

In 1939 P. Lévy [18] systematically extended the notions of expectation and variance to spherical random variables $X$ taking values in the torus $\mathbb{I}:=J-\pi, \pi$ l instead of the real line $\mathbb{R}$. Lévy proposed the definition

$$
V(X):=\inf _{a \in \mathbf{T}} \int_{\mathbf{T}}(x-a)^{2} P_{X}(d x)
$$

for the variance of $X$, and every number a $\in \mathbf{I}$ such that the infimum in the definition of $V(X)$ is attained he called an expectation of $X$. Thus $E(X)$ is defined as any $a_{0} \in \mathbf{T}$ satisfying the inequality

$$
\int_{\mathbf{T}}\left(x-a_{0}\right)^{2} P_{X}(d x) \leqslant \int_{\mathbf{T}}(x-a)^{2} P_{X}(d x)
$$

for all a $\in \mathbf{T}$.

In the following, generalizations of Kolmogorov's three-series theorem will be of central interest. For real random variables this theorem states that for any sequence $\left(x_{j}\right)_{j \geqslant 1}$ of random variables the series $\sum_{j \geqslant 1} x_{j}$ converges a.s. $\left(\sum_{j \geqslant 1} x_{j}<\infty\right.$ a.s. for abbreviation) iff the following condition holds:

There exists a number $c>0$ such that

$$
\text { (a) } \sum_{j \geqslant 1} P\left[\left|x_{j}\right|>c\right]<\infty \text {, }
$$




$$
\begin{aligned}
& \text { (b) } \sum_{j \geqslant 1} E\left(x_{j}{ }^{1}\left[\left|x_{j}\right| \leqslant c\right]\right)<\infty \text {, and } \\
& \text { (c) } \sum_{j \geqslant 1} v\left(x_{j}{ }^{1}\left[\left|x_{j}\right| \leqslant c\right]\right)<\infty .
\end{aligned}
$$

In the case of spherical random variables the necessary and sufficient condition for $\sum_{j \geqslant 1} x_{j}<\infty$ a.s. turns out to be

$$
\begin{aligned}
& \text { (c') } \sum_{j \leqslant 1} v\left(x_{j}\right)<\infty \text { and } \\
& \text { (b') } \sum_{j \geqslant 1} E\left(x_{j}\right)<\infty,
\end{aligned}
$$

where ( $\left.b^{\prime}\right)$ has to be read in the sense that $\sum_{j \geqslant 1} E\left(X_{j}\right)<\infty$

for any choice of expectations $E\left(X_{j}\right)$ of $X_{j}$ or for some suitable choice of $E\left(X_{j}\right)$. See Lévy [18] and Bartfai [1].

It is the nonuniqueness of the expectation in this case which yields the appropriate extension of the concept to arbitrary compact groups.

\section{BASIC NOTIONS}

Before entering the subject proper a review of some basic notions from probability theory on a topological group seems to be in order. For details in the locally compact case the reader is referred to Heyer [14]. 
Let $G$ be a (completely regular) topological group with unit element e. By $\boldsymbol{\mu}^{\mathrm{b}}(G)$ we denote the set of all bounded Radon measures $\mu$ on $G$ in the sense that $\mu$ is a linear functional on the space $\varphi^{b}(G)$ of bounded continuous functions on $G$ possessing the following property: For every $\varepsilon>0$ there exists a compact subset $C_{\varepsilon}$ of $G$ such that $|\mu(g)| \leqslant \varepsilon$ for all $g \varepsilon \varphi^{b}(G)$ with $|g| \leqslant 1$ and $g\left(C_{\varepsilon}\right)=0$. Clearly, every Radon measure on $G$ can be considered as a bounded regular borel measure on the Borel-oalgebra $f(G)$ of $G$. In $\mu^{b}(G)$ we introduce the weak topology $\sigma_{W}$. In fact, the mapping $(\mu, \nu) \rightarrow \mu * \nu$ from $\mu^{b}(G) \times \mu^{b}(G)$ into $\mu^{b}(G)$ is continuous on norm bounded subsets. $\mu_{+}^{b}(G)$ is separable and metrizable iff $G$ is separable and metrizable.

If $G$ is a locally compact group, then there exists a left (or right) Haar measure $\omega:=\omega_{G}$ on $G$. This measure is unique up to a positive multiplicative constant. $\omega$ is $\sigma$-finite, and it is bounded iff $G$ is compact. On the subsemigroup $\mu^{1}(G)$ of all probability measures on $G$ the weak topology $\mathcal{}_{w}$ coincides with the vague topology $\tau_{\mathrm{v}}$ defined as the topology of pointwise convergence on the space $\mathcal{K}(G)$ of continuous functions on $G$ with compact support. $\mu^{1}(G)$ is compact or locally compact iff $G$ is compact. For any compact subgroup $H$ of $G, \omega_{H}$ denotes the normed Haar measure of $H$ defined as the (both sided) H-invariant measure in $\mu^{1}(G)$ having $H$ as its support. The normed Haar measures of compact subgroups are exactly the idempotents of $\boldsymbol{\mu}^{1}(G)$ and also of $\mu_{+}^{b}(G)$. 
For every $x \in G$ the symbol $\varepsilon_{x}$ denotes the Dirac measure in $x$. The set of all Dirac measures on $G$ will be abbreviated by $D(G)$.

\section{THE THREE-SERIES THEOREM IN BANACH SPACES}

We are given a Bernoulli sequence on some probability space $(\Omega, O, P)$, that is a sequence $\left(\varepsilon_{j}\right)_{j \geqslant 1}$ of independent random variables $\varepsilon_{j}$ such that $P\left[\varepsilon_{j}=-1\right]=P\left[\varepsilon_{j}=+1\right]=\frac{1}{5}$ for all $j \geqslant 1$.

Let $E$ be a Banach space, $p \in[1,2]$ and $q \in[2, \infty]$. We start with the following

DEFINITION. $E$ is said to be of (Rademacher) type $p$ if there exists a constant $K>0$ such that

$$
E\left\|\sum_{j=1}^{n} \varepsilon_{j} x_{j}\right\|^{P} \leqslant k \sum_{j=1}^{n}\left\|x_{j}\right\|^{P}
$$

for all $n \geqslant 1$ and all sequences $\left\{x_{1}, \ldots, x_{n}\right\}$ in $E$.

Analoguously, $E$ is said to be of (Rademacher) cotype $q$ if there exists a constant $k>0$ such that

$$
E\left\|\sum_{j=1}^{n} \varepsilon_{j} x_{j}\right\|^{q} \geqslant k \sum_{j=1}^{n}\left\|x_{j}\right\|^{q}
$$

for all $n \geqslant 1^{\circ}$ and all sequences $\left\{x_{1}, \ldots, x_{n}\right\}$ in $E$.

Clearly, every Banach space is of type 1 and of cotype $\infty$. For any measure space $(S, \Sigma, \mu)$ and $p \in[1,2]$ the space $L^{P}\left(S, \sum, \mu\right)$ is 
of type $2 \wedge \mathrm{p}$ and of cotype $2 \vee \mathrm{p}$.

We are now considering sequences $\left(x_{j}\right)_{j \geqslant 1}$ of independent random variables taking values in a separable Banach space $E$. The norm closed unit ball of $E$ will be denoted by $B$. If $X$ is an E-valued random variable and $C$ a set in the Borel-o-algebra $\mathcal{F}_{(E)}$, then $X .1_{C}$ is defined to be equal to $X$ on $C$ and to 0 otherwise. If $X$ is P-integrable, $E(X)$ will denote the expectation of $X$.

Given a sequence $\left(X_{j}\right)_{j \geqslant 1}$ of independent E-valued random variables we shall study the a.s. convergence of the series $\sum_{j \geqslant 1} x_{j}$.

Let $p \in[1, \infty[$.

DEFINITION. A separable Banach space $E$ is said to admit the Kolmogorov property of order $P\left(K P_{P}\right)$ if for every sequence $\left(X_{j}\right)_{j \geqslant 1}$ of independent E-valued random variables, the series $\sum_{j \geqslant 1} x_{j}$ converges a.s. whenever there exists a constant $c>0$ such that

$$
\sum_{j \geqslant 1} P\left[\left\|x_{j}\right\|<c\right]<\infty
$$

$$
\sum_{j \geqslant 1} E\left(X_{j} \cdot 1_{C B}\right) \text { is summable in } E \text {, and }
$$

$$
\sum_{j \geqslant 1} E\left(\left\|X_{j} \cdot 1_{c B}-E\left(X_{j} \cdot 1_{c B}\right)\right\|^{P}\right)<\infty .
$$

$E$ is said to admit the strong Kolmogorov property of order $p$ $\left(S K P_{p}\right)$ if for every sequence $\left(X_{j}\right)_{j \geqslant 1}$ of independent E-valued random variables, the a.s. convergence of the series $\sum_{j \geqslant 1} x_{j}$ is equivalent 
to (S1) through (S3) for some $c>0$.

THEOREM 3.1. A separable Banach space $E$ admits $\left(K P_{p}\right)$ for $p \in[1,2]$ iff $E$ is of type $p$.

Concerning the PROOF of the theorem we note that the implication "—" is essentially known. See Woyczyński [35], p. 431 for the special case $p=2$. The inverse implication follows directly from HoffmannJorgensen [15], p. 116.

THEOREM 3.2. A separable Banach space $E$ admits $\left(\mathrm{SKP}_{2}\right)$ iff $E$ is a Hilbert space.

The PROOF of this result is based on a famous theorem of Kwapien's on the type-cotype characterization of Hilbert spaces. See Kwapien [17].

4. MOMENT CONDITIONS IN THE CASE OF AN ABELIAN LOCALLY COMPACT GROUP

We start by restating the classical three-series theorem in terms of characters of the additive group of the real line $\mathbb{R}$. For any $\mathbb{R}$-valued random variable $X$ on a probability space $(\Omega, O, P$, and any character $x$ of $\mathbb{R}$ of the form $x \rightarrow x(x)=x_{y}(x):=e^{\text {iyx }}$ for some $y \in \mathbb{R}$ we define the $\mathrm{T}$-valued random variable $\mathrm{Y}:=\mathrm{y}_{\mathrm{O}} \mathrm{Z}$, where $y_{0}:=\frac{c}{\pi}$ for some $c>0$, and $z$ denotes the principal branch of the $\mathbf{T}$-valued random variable $\exp \left(\frac{i}{y_{0}} X\right)=\exp \left(i \frac{\pi}{c} X\right)=\exp \left(i \pi \frac{X}{c}\right)$. Noting that with this definition of $Z,[X \neq Z]=[-c<X \leqslant c]$, we 
arrive at the following version of Kolmogorov's result.

THEOREM 4.1. For any sequence $\left(X_{j}\right)_{j \geqslant 1}$ of independent $\mathbb{R}$-valued random variables the following statements are equivalent:

(i) $\sum_{j \geqslant 1} x_{j}<\infty \quad$ a.s.

(ii) There exists a neighborhood $U:=]-c, c]$ of $\circ$ such that

(a) $\sum_{j \geqslant 1} P\left[X_{j} \in[U]<\infty\right.$,

(b) $\sum_{j \geqslant 1} E\left(Y_{j}\right)<\infty$, and

(c) $\sum_{j \geqslant 1} V\left(Y_{j}\right)<\infty$.

Using the above device the convergence conditions (a) through (c) of (ii) can be restated as

$$
\begin{aligned}
& \text { (a') } \sum_{j \geqslant 1} P\left[x_{j} \in[U]<\infty,\right. \\
& \text { (b') } \sum_{j \geqslant 1} E\left(\log x \circ x_{j}\right)<\infty, \text { and } \\
& \text { (c') } \sum_{j \geqslant 1} V\left(\log x \circ x_{j}\right)<\infty,
\end{aligned}
$$

the latter two holding for every character $x$ of $\mathbb{R}$. Here the $\mathbf{T}$-valued random variable of the form $\log x \circ \mathrm{X}$ is defined as 
iZ where $Z$ denotes the principal branch of the $\mathbf{T}$-random variable $\exp (i x \circ X)$.

Now, let $G$ be an Abelian locally compact group which we write multiplicatively with unit element $e$. We suppose that $G$ is second countable. If we denote the character group of $G$ by $G^{\wedge}$ we have the following result of R.P. Pakshirajan [27].

THEOREM 4.2. For every sequence $\left(X_{j}\right)_{j \geqslant 1}$ of independent G-valued random variables the following statements are equivalent:

(i) $\quad \prod_{j \geqslant 1} x_{j}<\infty \quad$ a.s.

(ii) (a) Given any (compact) neighborhood $U$ of $e$, the series $\sum_{j \geqslant 1} P\left[X_{j} \in[U]\right.$ converges.

(b) $\sum_{j \geqslant 1} E\left(\log x \circ x_{j}\right)<\infty$,

(c) $\sum_{j \geqslant 1} V\left(\log x \circ x_{j}\right)<\infty$,

the latter two conditions holding for every $x \in G^{\wedge}$, and the $\mathbf{T}$-random variables log $x_{j} x_{j}$ defined as indicated above.

The PROOF of this theorem relies on a few auxiliary results.

LEMMA 1. For every $x \in G^{\wedge}$ the following statements are equivalent: 
(i) $\quad \prod_{j \geqslant 1} x \circ x_{j}<\infty \quad$ a.s.

(ii) $\prod_{j \geqslant 1} E\left(x \circ x_{j}\right)<\infty$.

LEMMA 2. For every $x \in G^{\wedge}$ the following two sets of conditions are equivalent:

(i) $\prod_{j \geqslant 1} E\left(x \circ x_{j}\right)<\infty$ and $\sum_{j \geqslant 1} V\left(x \circ x_{j}\right)<\infty$.

(ii) $\sum_{j \geqslant 1} E\left(z_{j}\right)<\infty$ and $\sum_{j \geqslant 1} V\left(z_{j}\right)<\infty$.

LEMMA 3. The following statements are equivalent:

(i) $\quad \prod_{j \geqslant 1} x_{j}<\infty \quad$ a.s.

(ii) (a) There exists a (compact) neighborhood $U$ of $e$ such that $\sum_{j \geqslant 1} P\left[X_{j} \in[U]<\infty\right.$.

(в) $\prod_{j \leqslant 1} x^{\circ}<X_{j}<$ a.s. for all $x \in G^{\wedge}$.

A more condensed version of Kolmogorov's theorem reads as follows.

THEOREM 4.3. Let $G$ be a second countable Abelian locally compact group. Then for any sequence $\left(X_{j}\right)_{j \geqslant 1}$ of independent G-valued random variables the following conditions are equivalent: 
(i)

$$
\prod_{j \geqslant 1} x_{j}<\infty
$$

$$
\prod_{j \geqslant 1} x \circ x_{j}<\infty
$$

For the PROOF as given in Pakshirajan [27] one notes this:

The implication $(i) \Rightarrow$ (ii) follows directly from the continuity of

the characters $x \in G \hat{0}$

The implication $(i i) \rightarrow(i)$ has to be shown for compact and for discrete Abelian groups first, before the structure theorem for locally compact Abelian groups can be applied.

\section{AN AXIOMATIC APPROACH TO EXPECTATIONS AND VARIANCES}

Let $G$ be a compact group and let $\mathcal{N}$ be a subsemigroup of $\boldsymbol{\mu}^{1}(G)$ such that $D(G) \subset \mathcal{N}$.

DEFINITION. $\mathcal{N}$ is said to admit an expectation $E$ if $E$ is a continuous semigroup homomorphism $\mathcal{S} \rightarrow G$ satisfying the following conditions:

(E 1) $E\left(\varepsilon_{x}\right)=x$ for all $x \in G$.

(E 2) $E(\mu)=e$ for all symmetric measures $\mu \in \mathcal{N}$.

REMARK. If $\boldsymbol{N}$ admits an expectation, then $\boldsymbol{N}$ does not contain any nontrivial idempotent of $\mu^{1}(G)$. In fact, let $\mu$ be an idempotent of $\Omega^{1}(G)$ of the form $\mu=\omega_{H}$ for some compact subgroup $H$ 
of $G$ such that $\mu \in \mathcal{N}$. Then $\omega_{H}=\varepsilon x{ }^{\star} \omega_{H}$ for all $x \in H$, whence $E\left(\omega_{H}\right)=E\left(\varepsilon_{x} \star \omega_{H}\right)=E\left(\varepsilon_{x}\right) E\left(\omega_{H}\right)=x E\left(\omega_{H}\right)$, and consequently $E\left(\varepsilon_{X}\right)=x=e$, which shows $H=\{e\}$.

From this remark it becomes clear that for the subsequent discussion we shall assume that the subsemigroups $\mathcal{N}$ with $D(G) \subset \mathcal{N}$ are neighborhoods of $\varepsilon_{e}$ in $\mu^{1}(G)$.

REMARK. Under this additional assumption $G$ is necessarily a Lie group (of dimension $\mathrm{p} \geqslant 0$ ).

For this one has to show that there exists a neighborhood of $e$ without any nontrivial subgroup of $G$. Suppose that for every neighborhood of $e$ there exists a nontrivial and hence a nontrivial compact subgroup $K$. Then $\omega_{K}$ is a neighborhood of $\varepsilon_{e}$ in $\mu^{1}(G)$, thus $\omega_{K} \in \mathcal{N}$ and consequently $K=\{e\}$. But this contradicts the as sumption.

DEFINITION. A compact group $G$ admits an expectation if there exists a subsemigroup $\mathcal{N}$ of $\boldsymbol{M}^{1}(G)$ with $\theta(G) \subset \mathcal{N}$ which is a neigh-. borhood of $\varepsilon_{e}$ in $\mu^{1}(G)$ such that $r$ admits an expectation.

The following result is due to V.M. Maksimov [22], [24].

THEOREM 5.1. For any compact group $G$ the following statements are equivalent:

(i) G admits an expectation.

(ii) There exist integers $p, q, r \geqslant 0$ such that $G \cong \operatorname{SU}(2) p \times \mathbb{T}^{q} \times \mathbb{Z}_{2}^{r}$, 
where SU(2) denotes the special linear group of dimension 2 , $\mathbb{I}$ the 1-dimensional torus and $\mathbb{Z}_{2}$ the 2-element group.

Concerning the PROOF of this existence theorem we shall sketch the ideas of both implications.

(1) Compact groups admitting expectations are necessarily of the form $G=\operatorname{SU}(2, p) \times \mathbb{T}(q) \times \mathbb{Z}_{2}(r)$ with $S U(2, p):=\operatorname{SU}(2) P$, $\mathbf{T}(q):=\mathbf{T}^{q}$ and $\mathbb{Z}_{2}(r):=\mathbb{z}_{2}^{r}$.

Let $\Sigma:=\sum(G)$ denote the system of irreducible (unitary continuous) representations of $G$. For every $\mu \in \mu^{1}(G)$ and each $\sigma \in \Sigma$ the Fourier transform of $\mu$ corresponding to the class $\sigma$ with representative $D^{(\sigma)}$ is defined by

$$
D^{(\sigma)}(\mu):=\int_{G} D^{(\sigma)}(x) \mu(d x) \in M(d(\sigma), \mathbb{C})
$$

where $\mathrm{d}(\sigma):=\mathrm{d}\left(D^{(\sigma)}\right)$ is the dimension of the representing (Hilbert) space $\mathcal{H}(\sigma):=H_{C}\left(D^{(\sigma)}\right)$ of $\sigma$ or $D^{(\sigma)}$.

For any subset $\boldsymbol{r}$ of $\boldsymbol{\mu}^{1}(G)$ we consider the subset $D^{(\sigma)}(\boldsymbol{r})$ of $M(d(\sigma), \mathbb{C})$. By the Peter-Weyl theorem the set

$\mu_{0}^{1}(G):=\left\{\mu \in \mu^{1}(G): D^{(\sigma)}(\mu) \neq 0\right.$ for finitely many $\left.\sigma \in \sum\right\}$

is dense in $\mu^{1}(G)$. It is therefore quite reasonable to deal with representations of the form 


$$
D^{N}:=\sum_{i=1}^{N} \oplus D_{D}\left(\sigma_{i}\right) .
$$

We note that $D^{N}(\mathcal{N})$ is a subsemigroup of $M\left(d\left(D^{N}\right), \mathbb{C}\right)$ whenever $\mathbb{R}$ is a subsemigroup of $\boldsymbol{M}^{1}(G)$.

STEP I. Every semigroup homomorphism $\varphi$ from $\mathcal{N}$ into some group $G_{1}$ induces a semigroup homomorphism $\bar{\varphi}$ from $D^{N}(\mathcal{N})$ into $G_{1}$.

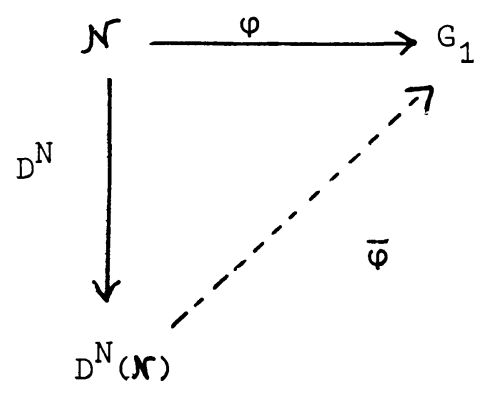

For every $\sigma \in \sum$ and $D^{(\sigma)} \in \sigma$ let $\mathcal{A}\left(D^{(\sigma)}\right)$ be the matrix algebra generated by the set $\left\{D^{(\sigma)}(x): x \in G\right\}$. Then $f\left(D^{(\sigma)}\right)$ is simple and thus isomorphic to one of the algebras $\operatorname{GL}(d(\sigma), \mathbb{R}), \operatorname{GL}(d(\sigma), \mathbb{C})$ or $G L(d(\sigma), \mathbb{H})$. Analoguously one defines $A\left(D^{N}\right)$.

STEP II. Every semigroup homomorphism $\bar{\varphi}$ from $D^{N}(\mathbb{N})$ into a group $G_{1}$ can be extended to a group homomorphism $\overline{\bar{\varphi}}$ from the group (generated by) $\&\left(D^{N}\right)$ into $G_{1}$. 


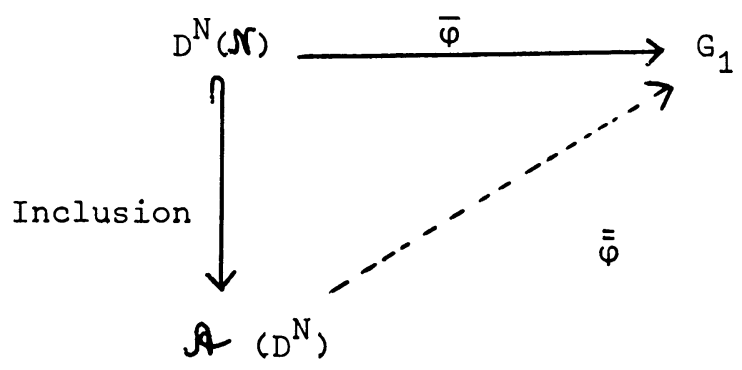

STEP III. It turns out that the compact group $G$ is a direct product of homomorphic images of the groups $G L(d, \mathbb{R}), G L(d, \mathbb{C})$ or $G L(d, \mathbb{H})$ into $G$. But such homomorphisms are known from the general theory of determinants over fields.

(2) Determination of expectations on the groups SU(2), $\mathbf{T}$ and $\mathbf{z}_{2}$. (2a) We first consider the group

$\operatorname{SU}(2)=\left\{\left(\begin{array}{cc}\alpha & \beta \\ -\bar{\beta} & \bar{\alpha}\end{array}\right): \quad|\alpha|^{2}+|\beta|^{2}=1\right\}$

and define the semigroup

$\mathcal{N}:=\left\{\mu \in \mu^{1}(\operatorname{SU}(2)): \operatorname{det} \int_{S U(2)}\left(-\bar{B} \frac{\beta}{\alpha}\right) d \mu \neq 0\right\}$.

For every $\mu \in \mathcal{N}$ we introduce

$$
E(\mu):=\frac{1}{\lambda(\mu)} \int_{S U(2)}\left(\begin{array}{ll}
\alpha & \frac{\beta}{\beta}
\end{array}\right) d_{\mu},
$$

where $\lambda(\mu)$ is determined by the condition $\operatorname{det} E(\mu)=1$. Then $E$ is the unique expectation of $S U(2)$. 


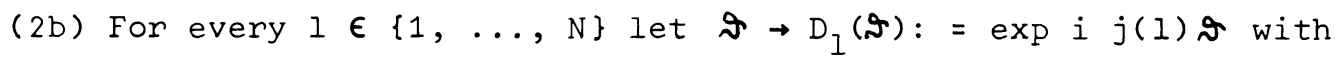
$j(1) \in \mathbb{Z}$ be a character of $\mathbf{T}$. Given a measure $\mu \in \boldsymbol{\mu}^{1}(\mathbf{T})$ we obtain, again for every $I \in\{1, \ldots, N\}$ the 1 -th Fourier coefficient of $\mu$ in the form

$$
D_{1}(\mu)=r_{1} \exp \text { isll with } r_{1} \in \mathbb{R}_{+}, g^{(1)} \in[0,2 \pi[.
$$

Let $\mathcal{N}:=\left\{\mu \in \mu^{1}(\mathbb{I}): D_{1}(\mu), \ldots, D_{1}(\mu) \neq 0\right\}$. Then for every $\mu \in \mathcal{N}$ we define an expectation $E$ of $\mathbf{T}$ by

$$
E(\mu):=\left\langle\left(N^{(1)}, \ldots, N^{(N)}\right),\left(\gamma_{1}, \ldots, \gamma_{N}\right)\right\rangle(\bmod 2 \pi),
$$

where $\left(\gamma_{1} \ldots, \gamma_{N}\right)$ denotes any vector of $\mathbf{z}^{N}$ satisfying

$$
\left\langle(j(1), \ldots, j(N)),\left(\gamma_{1}, \ldots, \gamma_{N}\right)\right\rangle=1
$$

(2c) The group $\mathbb{z}_{2}=\{e, x\}$ admits a unique expectation $E$ defined by

$$
E(\mu):= \begin{cases}e & \text { if } \mu(\{e\})>\mu(\{x\}) \\ x & \text { otherwise }\end{cases}
$$

whenever $\mu \in \boldsymbol{M}^{1}\left(\mathbb{Z}_{2}\right)$.

Now we proceed to second moments.

DEFINITION. $\mathcal{N}$ is said to admit a variance $V$ if $V$ is a non- 
constant continuous semigroup homomorphism $\boldsymbol{N} \rightarrow \mathbb{R}_{+}$satisfying the following conditions:

$\begin{array}{ll}\text { (V1) } V(\mathcal{N}) & \subset \quad[0,1] . \\ \text { (V2) } V(\mu) & =1 \text { iff } \mu \in D(G) .\end{array}$

Any nonconstant continuous semigroup homomorphism $\mathbb{N} \rightarrow \mathbb{R}_{+}$is called a weak variance on $\mathcal{N}$. Variances and weak variances on $\mathcal{N}:=\boldsymbol{\mu}^{1}(G)$ are called variances and weak variances for $G$. In the first case we also say that $G$ admits a variance if there exists a variance on $\mu^{1}(G)$.

REMARK. A weak variance $V$ on $\mathcal{N}$ is a variance iff $V(\mu)=0$ for all nontrivial idempotents $\mu$ of $\mu^{1}(G)$ in $\boldsymbol{N}$. Variances have been first defined by V.M. Maksimov in [19] and [20]. For a detailed presentation of the theory we recommend Heyer [14], 2.4 .

THEOREM 5.2. Let $G$ be a compact group, $\sigma_{1}, \ldots, \sigma_{\mathrm{N}} \in \sum$ with representatives $D^{\left(\sigma_{1}\right)}, \ldots, D^{\left(\sigma_{N}\right)}$ and $k_{1}, \ldots, k_{N} \in \mathbb{R}_{+}^{\star}$. Then the mapping

$$
\mu \rightarrow V(\mu):=\prod_{i=1}^{N}\left|\operatorname{det} D^{\left(\sigma_{i}\right)}(\mu)\right|^{k_{i}}
$$

from $\operatorname{su}^{1}(G)$ into $\mathbb{R}_{+}$is a weak variance for $G$. Moreover, the following statements are equivalent:

(i) $\quad V$ is a variance for $G$.

(ii) The smallest set $\left[\sigma_{1}, \ldots, \sigma_{N}\right]$ closed under products and 
conjugates and containing $\left\{\sigma_{1}, \ldots, \sigma_{N}\right\}$ equals $\sum$.

(iii) $D^{N}=\sum_{i=1}^{N} \oplus D^{\left(\sigma_{i}\right)}$ is faithful.

THEOREM 5.3. For any compact group $G$ the following statements are equivalent:

(i) $G$ admits a variance.

(ii) $G$ is a Lie group (finite or infinite).

The PROOF of this result involves the general form of weak variances on $\mu^{\mathrm{b}}(G)$.

STEP I. Let $A$ be a simple algebra over $\mathbb{R}$ with unit element and $\varphi$ a nonconstant continuous homomorphism from the multiplicative semigroup $A$ into $\mathbb{R}_{+}$. Then there exist a representation $D$ of $A$ and a $k \in \mathbb{R}_{+}^{*}$ such that

$$
\varphi(a)=|\operatorname{det} D(a)|^{k}
$$

for all a $\in$ A.

STEP II. Let $A:=\sum_{i \in I}^{\oplus} A_{i}$ be the Hilbert sum of a family $\left(A_{i}\right)_{i \in I}$ of simple algebras over $\mathbb{R}$ with unit and let $\varphi$ be a nonconstant continuous semigroup homomorphism form $A$ into $\mathbb{R}_{+}$. Then there exist a finite subset $I_{0}$ of $I$ and for every $i \in I_{\circ}$ a representation $D_{i}$ of $A_{i}$ as well as a $k_{i} \in \mathbb{R}_{+}^{*}$ such that 


$$
\varphi(a)=\prod_{i \in I_{0}}\left|\operatorname{det} D_{i}\left(a_{i}\right)\right|^{k}
$$

for all a: $=\left(a_{i}\right)_{i \in I} \in A$.

Application of this fact to the algebra $A:=I_{\mathbb{R}}^{2}(G, \omega)$ for our compact group $G$ (and its Haar measure $\omega$ ) which by the Peter-Weyl theorem admits the decomposition

$$
I_{\mathbb{R}}^{2}(G, \omega)=\sum_{\sigma \in \sum}^{\oplus} L_{\sigma},
$$

where for every $\sigma \in \sum, L_{\sigma}$ is the simple ideal

$$
L_{\sigma}:=\left\{x^{(\sigma)} \star f: f \in L_{\mathbb{R}}^{2}(G, \omega)\right\}
$$

with $x^{(\sigma)}:=\operatorname{tr} D^{(\sigma)}$ for $D^{(\sigma)} \in \sigma \in \sum$, yields

STEP III. Any nonconstant continuous semigroup homomorphism $\varphi$ form $\mu^{b}(G)$ into $\mathbb{R}_{+}$is of the form

$$
\varphi(\mu)=\prod_{i=1}^{N}\left|\operatorname{det} D^{\left(\sigma_{i}\right)(\mu)}\right|^{k_{i}}
$$

for all $\mu \in \mu^{b}(G)$, where $\sigma_{1}, \ldots, \sigma_{N} \in \sum$ and $k_{1}, \ldots, k_{N} \in \mathbb{R}_{+}^{*}$. 


\section{A GENERAL THREE-SERIES THEOREM}

Let $G$ be a compact group. For any sequence $\left(\mu_{j}\right)_{j \geqslant 1}$ in $\mu^{1}(G)$ we consider the corresponding sequences $\left(\nu_{k, n}\right)_{n>k}$ of partial convolution products $\nu_{k, n}:=\mu_{k+1} \star \ldots \ldots * \mu_{n}$.

DEFINITION. The sequence $\left(\mu_{j}\right)_{j \geqslant 1}$ is said to be composition convergent if for every $k \geqslant 0$ the sequence $\left(\nu_{k, n}\right)_{n>k}$ converges. In the case of composition convergence one has $\lim _{n \rightarrow \infty} \nu_{k, n}=\nu^{(k)}$ for every $k \geqslant 0$, and $\lim _{k \rightarrow \infty} v^{(k)}=v^{(\infty)}=\omega_{H}$ for some compact subgroup $H$ of $G$. $\mathrm{H}$ is called the basis of $\left(\mu_{j}\right)_{j \geqslant 1}$. In fact, $\mathrm{H}$ is the maximal compact subgroup of $G$ with the property $\nu^{(k)}=\nu^{(k)} * \varepsilon_{x}$ for all $x \in H$ or $v^{(k)}=v^{(k)} * \omega_{H}$ whenever $k \geqslant 0$.

THEOREM 6.1. Let $G$ be a compact group admitting an ispeacation $E$ and a variance $V$ on some subsemigroup $\mathcal{N}$ of $\mu^{1}(G)$ ? every sequence $\left(\mu_{j}\right)_{j \geqslant 1}$ in $\boldsymbol{N}$ the following statements are equivalent:

(i) $\left(\mu_{j}\right)_{j \geqslant 1}$ is composition convergent with basis $\{e\}$.

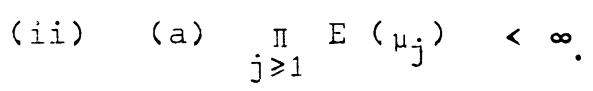

(b) $\sum_{j \geqslant 1}\left(1-V\left(\mu_{j}\right)\right)<\infty$.

For the PROOF of the theorem see Maksimov [24]. 
DISCUSSION. The preceding result generalizes the classical three-series theorem to compact (Lie)groups, since in $\mathbb{R}$ the wellknown equivalence theorem states that for any sequence $\left(x_{j}\right)_{j \geqslant 1}$ of independent random variables, a.s. convergence and convergence in distribution of $\sum_{j \geqslant 1} x_{j}$, i.e. of the sequence $\left(\sum_{j=1}^{n} x_{j}\right)_{n \geqslant 1}$ of $n-t h$ partial sums, are equivalent.

This equivalence holds for random variables taking values in a locally compact group $G$ iff $G$ has no nontrivial compact subgroups. (See [14], 2.2) Therefore, in the case of a compact group $G$ one is motivated to replace the a.s. convergence of $\prod_{j \geqslant 1} x_{j}$ by the $\boldsymbol{\zeta}_{\omega}$-convergence of the sequence $\left(\nu_{n}\right)_{n \geqslant 1}$ of $n$-th partial products $\nu_{n}:=\nu_{0, n}$.

In the case of a separable Banach space the equivalence theorem holds without any restriction. One recalls the ItồNisio theorem as quoted in Woyczyński [35], p. 274.

\section{THE SPECIAL CASES OF FINITE GROUPS AND LIE GROUPS}

Here we shall discuss versions of the three-series theorem for general Lie groups including finite groups.

First of all we take up the case of a finite group $G$ of order $p$ of the form $G=\left\{x_{1}, \ldots, x_{p}\right\}$, where $x_{1}$ denotes the neutral element e of $G$.

Let $\left(X_{j}\right)_{j \geqslant 1}$ be a sequence of independent $G$-valued random variables on a probability space $(\Omega, O, P)$. As before we form 
the corresponding sequence $\left(Y_{n}\right)_{n \geqslant 1}$ of $G$-valued random variables $Y_{n}:=X_{1} \ldots x_{n}$.

Clearly $\left(Y_{n}\right)_{n \geqslant 1}$ converges a.s. if for $P-a . a . \omega \in \Omega$ there exists an $j(\omega) \geqslant 1$ such that $x_{j}(\omega)=e$ for all $j \geqslant j(\omega)$. Thus, for the set

$$
c:=\bigcup_{l \geqslant 1} \bigcap_{j \geqslant 1}\left[x_{j}=e\right]
$$

of points of convergence of $\left(Y_{n}\right)_{n \geqslant 1}$ we get $P(C)=0$ or 1 . It follows that the a.s. convergence of $\left(Y_{n}\right)_{n \geqslant 1}$ is equivalent to the inequality

$$
P\left(\bigcap_{j \geqslant j}\left[x_{j}=e\right]\right)>0 \text { for some } j_{0} \geqslant 1 \text {. }
$$

Since for every $j \geqslant 1$ the distribution $\mu_{j}:=P_{X_{j}}$ of $x_{j}$ is of the form $\mu_{j}=\alpha_{1}{ }^{(j)} \varepsilon_{x_{1}}+\ldots+\alpha_{p}{ }^{(j)} \varepsilon_{x_{p}}$, the a.s. convergence of $\left(Y_{n}\right)_{n \geqslant 1}$ is in fact equivalent to the inequality

$$
\prod_{j \geqslant j} \alpha_{1}(j)>0 \text { for some } j_{0} \geqslant 1 \text {. }
$$

Wịth the notation $\alpha^{(j)}:=\max \left(\alpha_{1}{ }^{(j)}, \ldots, \alpha_{p}{ }^{(j)}\right.$ ) for all $j \geqslant 1$, V.M. Maksimov in [19] proves the

THEOREM 7.1. For any sequence $\left(X_{j}\right)_{j \geqslant 1}$ of $G$-valued random variables $x_{j}$ with distribution $\mu_{j}$ of the form 


$$
\mu_{j}=\alpha_{1}^{(j)} \varepsilon_{x_{1}}+\ldots+\alpha_{p}^{(j)} \varepsilon_{x_{p}}
$$

the following statements are equivalent:

(i) $\sum_{j \geqslant 1}\left(1-V\left(\mu_{j}\right)\right)<\infty$ in the sense that

$$
\prod_{j \geqslant j_{0}} V\left(\mu_{j}\right)>0 \quad \text { for some } j_{0} \geqslant 1 \text {. }
$$

(ii) $\prod_{j \geqslant 1} \alpha^{(j)}>0$.

As a direct consequence of this result one obtains the three-series

THEOREM 7.2. For any sequence $\left(X_{j}\right)_{j \geqslant 1}$ of independent G-valued random variables $x_{j}$ with distribution $\mu_{j}$ the following statements are equivalent:

(i) $\left(Y_{n}\right)_{n \geqslant 1}$ converges a.s.

(ii) (a) $\sum_{j \geqslant 1}\left(1-V\left(\mu_{j}\right)\right)<\infty$.

(b) $\left(\mu_{j}\right)_{j \geqslant 1}$ converges to $\varepsilon_{e}$.

DISCUSSION (Comparison with the classical situation). Condition (ii) (a) corresponds to the classical condition

(A) $\sum_{j \geqslant 1} V\left(x_{j} 1_{U}\right)=E\left[x_{j} 1_{U}-E\left(x_{j}{ }^{1} U\right)\right]^{2}<\infty$ 
for the variances $V\left(X_{j}{ }^{1} U\right)$ of truncated variables $X_{j}{ }^{1} U$, $U$ denoting a neighborhood of 0 .

Condition (ii) (b) corresponds to the classical condition

(B) $\sum_{j \geqslant 1} E\left(X_{j} 1_{U}\right)<\infty$,

since from (A) and (B) follows that the variables $X_{j}{ }^{1}$ are concentrated at 0 .

It turns out that the third classical condition

$$
\text { (C) } \sum_{j \geqslant 1} P\left[X_{j} \in[U]<\infty\right.
$$

is redundant in the case of a finite group $G$. The corresponding condition (in terms of a nelghborhood $U$ of $e$ ) can be deduced from conditions (ii) (a) and (b). In fact, these conditions together with the above THEOREM yield

$$
\lim _{j \geqslant j} \alpha_{1}^{(j)}>\quad 0
$$

But $P\left[X_{j} \in[U] \leqslant 1-P\left[x_{j}=e\right]\right.$ implies

$\sum_{j \geqslant 1} P\left[x_{j} \in[U] \leqslant \sum_{j \geqslant 1}\left(1-P\left[x_{j}=e\right]\right)=\sum_{j \geqslant 1}\left(1-\alpha_{1}^{(j)}\right)<\infty\right.$,

whence condition (C). 
We proceed to the case of a lie group G.

Let $G$ be a Lie group of dimension $p \geqslant 1$ and let $\left(x_{j}\right)_{j \geqslant 1}$ be a sequence of independent G-valued random variables $X_{j}$ on $(\Omega, O, P$, with distribution $\mu_{j}$. We consider the sequence $\left(Y_{n}\right)_{n \geqslant 1}$ of $n-t h$ partial products $Y_{n}:=X_{1} \ldots X_{n}$. If for some neighborhood $U$ of e the series

$$
\sum_{j \geqslant 1} P\left[X_{j} \in[U]=\sum_{j \geqslant 1} \mu_{j}\left([U)=\sum_{j \geqslant 1}\left(1-\mu_{j}(U)\right)\right.\right.
$$

diverges, then $\left(Y_{n}\right)_{n \geqslant 1}$ diverges a.s. Since we are interested in studying the a.s. convergence of $\left(Y_{n}\right)_{n \geqslant 1}$ we may assume without loss of generality that

$$
x_{j}(\Omega) \subset U \text { for all } j \geqslant 1 \text {, }
$$

where $U$ can be chosen as a (local) coordinate neighborhood defining a local coordinate system of $G$. Such a coordinate neighborhood generates a connected component of $G$, whence we may also assume that $G$ is connected.

Let $\left\{x_{1}, \ldots, x_{p}\right\}$ denote a fixed local coordinate system with coordinate neighborhood $U$ of $e$. For every $x \in U$ the symbol $\tilde{x}$ will denote the coordinate vector $\left(x_{1}(x), \ldots, x_{p}(x)\right)$ of $x$ with respect to the system $\left\{x_{1}, \ldots, x_{p}\right\}$. The correspondence $x \rightarrow \tilde{x}$ is a continuous bijection from $U$ onto its image in $\mathbb{R}^{p}$. For every $j \geqslant 1$ the G-valued random variable $X_{j}$ induces an $\mathbb{R}^{P}$-valued random varia- 
ble $\tilde{x}_{j}($ on $(\Omega, \sigma, P))$.

The following significant result of V.M. Maksimov [23] is basic for his further studies (also in [21]) in establishing a three-series theorem for Lie group-valued random variables.

THEOREM 7.3. Let $G$ be a connected lie group of dimension $\mathrm{p} \geqslant 1$ and $\left\{\mathrm{x}_{1}, \ldots, \mathrm{x}_{\mathrm{p}}\right\}$ a local coordinate system with coordinate neighborhood $U$ of e. Let $\left(X_{j}\right)_{j \geqslant 1}$ be a sequence of independent $G$-valued random variables on $(\Omega, O, P)$ and $\left(Y_{n}\right)_{n \geqslant 1}$ the corresponding sequence of $\mathrm{n}$-th partial products. Then

(i) $\quad\left(Y_{n}\right)_{n \geqslant 1}$ converges a.s. if

(a) $E\left(\tilde{X}_{j}\right)=0$ for all $j \geqslant 1$, and

(b) $\sum_{j \geqslant 1}\left\|v\left(\tilde{X}_{j}\right)\right\|<\infty$.

(ii) $\left(Y_{n}\right)_{n \geqslant 1}$ diverges a.s. if

(a) $E\left(\tilde{x}_{j}\right)=0$ for all $j \geqslant 1$, and

(c) $\sum_{j \geqslant 1}\left\|v\left(\tilde{X}_{j}\right)\right\|=\infty$.

Here, $V\left(\tilde{X}_{j}\right)$ denotes the vector of the variances of the components of $\tilde{X}_{j}$, and $\|$. $\|$ stands for the Euclidean norm in $\mathbb{R}^{P}$.

DISCUSSION. Let $\left(x_{n}\right)_{n \geqslant 1}$ be a sequence in $U$ and let $\left(\tilde{x}_{n}\right)_{n \geqslant 1}$ denote the corresponding sequence of canonical coordinates. Then, for Abelian groups $G$, the product $\prod_{j \geqslant 1} x_{j}$ and the sum $\sum_{j \geqslant 1} \tilde{x}_{j}$ simultaneously converge or diverge. Moreover, it has been shown that in 
the case of a non-Abelian group $G$ this conclusion does not hold for any local coordinate system. Therefore we cannot expect the simultaneous convergence of $\prod_{j \geqslant 1} x_{j}$ and $\sum_{j \geqslant 1} \tilde{x}_{j}$. There is, however, a probabilistic version of this fact:

If $E\left(\tilde{X}_{j}\right)=0$ for all $j \geqslant 1$ and $\sum_{j \geqslant 1}\left\|V\left(\tilde{X}_{j}\right)\right\|<\infty$, then the sum $\sum_{j \geqslant 1} \tilde{x}_{j}$ converges a.s. On the other hand, by the THEOREM above, the product $\prod_{j \geqslant 1} X_{j}$ must also converge a.s. An analoguous statement holds true for simultaneous divergence a.s.

THEOREM 7.4. (One sided three-series theorem). Let $G$ be a connected Lie group of dimension $p \geqslant 1$ and $x_{1}, \ldots, x_{p}$ a local coordinate system with coordinate neighborhood $U$ of e. Let $\left(x_{j}\right) j \geqslant 1$ be a sequence of independent $G$-valued random variables on $(\Omega, O, P)$ and $\left(Y_{n}\right)_{n \geqslant 1}$ the corresponding sequence of $n$-th partial products. The sequence $\left(Y_{n}\right)_{n \geqslant 1}$ converges a.s. if

(a) $X_{j}(\Omega) \subset U$ for all $j \geqslant 1$.

(b) $\quad \sum_{j \geqslant 1}\left\|E\left(\tilde{x}_{j}\right)\right\|,<\infty$.

(c) $\sum_{j \geqslant 1}\left\|v\left(\tilde{x}_{j}\right)\right\|<\infty$.

MAKSIMOV'S CONJECTURE. Let $G$ be a connected Lis group and $U$ a coordinate neighborhood of e. For every sequence (.) $j \geqslant 1$ of independent G-valued random variables and the correspondil sequence $\left(Y_{n}\right)_{n \geqslant 1}$ of $n$-th partial products the following statei ents are equivalent: 
(i) $\quad\left(Y_{n}\right)_{n \geqslant 1}$ converges a.s.

(ii) (a) $\sum_{j \geqslant 1}\left\|E\left(\tilde{X}_{j}\right)\right\|<\infty$.

(b) $\sum_{j \geqslant 1}\left\|v\left(\tilde{x}_{j}\right)\right\|<\infty$.

(c) $\prod_{j \geqslant 1} x_{j}$ converges for any sequence

$$
\left(x_{j}\right)_{j \geqslant 1} \text { in } G \text { satisfying } \tilde{x}_{j}=E\left(\tilde{x}_{j}\right) \text { for all } j \geqslant 1 \text {. }
$$

REMARK. For Abelian Lie groups Maksimov's conjecture can be established with little effort.

8. HIGHER MOMENTS AND THE STRONG LAW OF LARGE NUMBERS ON A LIE GROUP

Moments of higher order of probability measures on a group have been studied for the first time by $Y$. Guivarc'h in [11]. They came up in the study of harmonic functions on locally compact groups and were applied to versions of the strong law of large numbers for groupvalued random variables.

Let $G$ be a locally compact group with a countable basis of its topology. We further assume that $G$ is (compactly) generated by a compact neighborhood $V$ of $e$ in the sense that $G$ coincides with the closed subgroup [V] generated by $\mathrm{V}$. For the moment let $\mathrm{V}$ be fixed. 
We introduce a mapping $\delta_{V}: G \rightarrow \mathbb{R}_{+}$by

$$
\delta_{V}(x):=\inf \left\{n \in \mathbb{N}^{\star}: \quad x \in V^{n}\right\}
$$

for all $x \in G$. Clearly, $\delta_{V}$ is subadditive, i.e.

$$
\delta_{V}(x y) \leqslant \delta_{V}(x)+\delta_{V}(y)
$$

whenever $x, y \in G$.

DEFINITION. A measure $\mu \in \mu^{1}(G)$ is said to admit a moment of order $\alpha \in \mathbb{R}_{+}^{*}$ if there exists a neighborhood $V$ of $e$ with $\left[V^{-}=G\right.$ such that

$$
\int_{G} \delta_{V}^{\alpha} d_{\mu}<\infty
$$

It can be shown that this definition is independent of the particular choice of the neighborhood $V$.

If $\mu \in \mu^{1}(G)$ admits a moment of order 1 and if $\lambda \in \operatorname{Hom}(G, \mathbb{R})$, then the integral

$$
\int_{G} \lambda(x) \mu(d x)
$$

exists. This observation motivates the following

DEFINITION. $\quad \mu \in M^{1}(G)$ is called centered if

(d) $\mu$ admits a moment of order 1 ,

(b) $\int \lambda d \mu=0$ for all $\lambda \in \operatorname{Hom}(G, \mathbb{R})$ 
The subsequent DISCUSSION shows that the notion of centering defined above generalizes the classical one.

Let $G^{*}:=\operatorname{Hom}(G, \mathbb{R})$ and $G^{\prime}$ the closed commutator subgroup of G. Then

$$
G^{*}=\left(G^{\prime} G^{\prime}\right)^{\star}=\left(\left(G / G^{\prime}\right) / K^{*},\right.
$$

where $K$ denotes the maximal compact subgroup of $G / G^{\prime}$. ConsequentIy $G^{*}$ is finite-dimensional and so is its dual $\bar{G}:=\left(G^{*}\right)^{\wedge}$. Let $x \rightarrow \bar{x}$ denote the canonical mapping from $G$ into $\bar{G}$ which maps $G$ onto a closed subgroup of $\bar{G}$ of the form $\mathbb{R}^{p} \times \mathbb{Z}^{q}$ with $p, q \geqslant 0$, such that $\left[\mathbb{R}^{\mathrm{P}} \times \mathbb{Z}^{\mathrm{q}}\right]^{-}=\overline{\mathrm{G}}$.

It is easy to see that for any $\mu \in \mu^{1}(G)$ admitting a moment of order $1, \mu$ is centered iff its image $\bar{\mu}$ under the canonical mapping $x \rightarrow \bar{x}$ is centered (in the classical sense).

As an application we cite the following profound

THEOREM 8.1. (Strong law of large numbers). Let $G$ be an amenable, connected Lie group, $\mu$ a measure in $\boldsymbol{S}^{1}(G)$ admitting moments of all orders, centered and satisfying $[\operatorname{supp}(\mu)]^{-}=G$. Then for any compact neighborhood $V$ of $e$ and for any sequence $\left(x_{j}\right)_{j \geqslant 1}$ of independent G-valued random variables (on a probability space $(\Omega, O$, P)) with common distribution $\mu$, the sequence $\left(Y_{n}\right)_{n \geqslant 1}$ of $n-t h$ partial products $Y_{n}:=X_{1} \cdot \ldots \cdot X_{n}$ satisfies the strong law of large numbers in the sense that 


$$
\lim _{n \rightarrow \infty} \frac{1}{n} \delta_{V} \quad Y_{n}=0[P]
$$

\section{FURTHER STUDIES ON MOMENTS OF PROBABILITY MEASURES}

Measures of dispersion such as variances for probabilities on the one-dimensional torus $\mathbf{T}$ have been first introduced by P. Lévy [18], and they have been applied to the study of limiting distributions also by P. Bartfai [1] .

Expectations and variances for probabilities on linear spaces are naturally defined as in the Euclidean space. First versions of the three-series theorem for Hilbert spaces or for the more general G-spaces are due to E. Mourier [25], [26]. For an expository presentation of the subject until 1970 see P. Ressel [28]. A discussion of the case of Hilbert space is also contained in the book [10] of I.I. Gihman and A.V. Skorohod.

Dispersions of probabilities on a sphere appear at an early stage in the work [7] of R.A. Fisher. Applications to problems of statistical estimation have been discussed by W. Uhlmann [32] and H. Vogt [34].

In the framework of the hyperbolic plane and space, expectations and variances can be introduced via derivatives of Fourier transforms of measures. A starting point was set by F.I. Karpelevich, V.N. Tutubalin and M.G. Shur in [16]. A different but similar approach is due 
to V.N. Tutubalin [31]. Applications to the central limit theorem indicated already in [16] have been made precise by J. Faraut [6]. They rely on the general theory of spherical functions (S. Helgason [12], N.J. Vilenkin [33]), especially for the Gelfand pair (SL (2, $\mathbb{R})$, SO $(2, \mathbb{R}))$, as discussed particularly in [29] by M. Sugiura, and their impact to probability theory on certain symmetric spaces (R. Gangolli [8], [9]).

The central limit theorem envisaged can be extended to all hyperbolic spaces. Its generalization to arbitrary Gelfand pairs seems to be a realistic goal.

There are various other approaches to dispersion measures of probabilities in a general setting.

The axiomatics of Section 5 extends to semigroups (see T. Byczkowski et al. in [2] and [3]). The semigroup $\mathbb{R}_{+}$is the basic structure of an approach to the notion of variance via generalized quadratic forms within the framework of hypergroups and generalized translation spaces (H. Chébli [4], [5], K. Trimèche [30]). Finally, we only mention the generalization of the Khintchine functional as a measure of dispersion and its importance to delphic theory. References and a few details are contained in the author's note [13] and in his monograph [14]. 


\section{REFERENCES}

[1] Bartfai, P.: Grenzwertsätze auf der Kreisperipherie und auf kompakten abelschen Gruppen. Studia Scient. Math. Hung. 1 (1966), 71-85

[2] Byczkowska, H.; Byczkowski, T.; Timoszyk, W.: Some properties of variance on compact groups. Bull. Acad. Polon. Sci. Sér. Sci. Math. Astronom. Phys. 20 (1972), $945-947$

[3] Byczkowski, T.: The notion of variance on compact topological semigroups.

Bull. Acad. Polon. Sci. Sér. Sci. Math. Astronom. Phys. 21 (1973), $945-950$

[4] Chébli, H.: Positivité des opérateurs de translation généralisée à un opérateur de Sturm-Liouville et quelques applications à l'analyse harmonique. Thesis presented at the University of Strasbourg (1974)

[5] Chébli, H.: Opérateurs de translation généralisée et semigroupes de convolution.

In: Théorie du potentiel et analyse harmonique. Lecture Notes in Math. 404, 35-59. Springer (1974)

[6] Faraut, J.: Dispersion d'une mesure de probabilité sur $\mathrm{SL}(2, \mathbb{R})$ biinvariante par SO $(2, \mathbb{R})$ et théorème de la limite centrale. Manuscript (1975).

[7] Fisher, R.A.: Dispersion on a sphere.

Proc. Roy. Soc. A 217 (1953), $295-305$

[8] Gangolli, R.: Isotropic infinitely divisible measures on symmetric spaces.

Acta Math. (Stockholm) 111 (1964), 213-246

[9] Gangolli, R.: Positive definite kernels on homogeneous spaces and certain stochastic processes related to Lévy's Brownian motion of several parameters.

Ann. Inst. Henri Poincaré Sect. $B_{2} \underline{3}$ (1967), $\frac{121}{121}-\frac{1}{225}$ 
[10] Gihman, I.I.; Skorohod, A.V.: The theory of stochastic processes $I$. Springer (1974)

[11] Guivarc'h, Y.: Lois des grands nombres et rayon spectral d'une marche aléatoire sur un groupe de Lie. In: Journées sur les marches aléatoires. Astérisque 74, 47-98. Société Mathématique de France (1980).

[12] Helgason, S.: Differential geometry and symmetric spaces. Academic Press (1962).

[13] Heyer, H.: Remarques sur une axiomatique de la variance. In: Les probabilités sur les structures algébriques, 177 - 189. Editions du Centre National de la Recherche Scientifique, Paris (1970).

[14] Heyer, H.: Probability measures on locally compact groups. Springer (1977)

[15] Hoffmann - Jorgensen, J.: Probability in Banach spaces. In: Ecole d'été de probabilitês de Saint flour VI 1976, Lecture Notes in Math. 598, 1 - 186. Springer (1977).

[16] Karpelevich, F.I.; Tutubalin, V.N.; Shur, M.G.: Limit theorems for the composition of distributions in the Lobachevsky plane and space.

Theory of Prob. and Appl. 4 (1959), 399-402.

[17] Kwapien, S.: Isomorphic characterizations of Hilbert spaces by orthogonal series with vector valued coefficients.

Sem. Maurey-Schivartz (1972/73), Exp. VIII.

[18] Lévy, P.: L'addition des variables aléatoires définies sur une circonférence.

Bull. Soc. Math. France 67 (1939), 1 - 41.

[19] Maksimov, V.M.: On the convergence of products of independent random variables taking on values from an arbitrary finite group. Theory of Prob. and Appl. 12 (1967), 619-637. 
[20] Maksimov, V.M.: A contribution to the theory of variance for probability distributions on compact groups. Soviet Math. Dokl. 11 (1970), 717 - 721 .

[21] Maksimov, V.M.: A convergence property of products of independent random variables on compact Lie groups. Math. USSR Sbornik 11 (1970), 423 - 440

[22] Maksimov, V.M.: Mathematical expectations for probability dis. tributions on compact groups and their applications.

Soviet Math. Dokl. $13(1972), 416$ - 419

[23] Maksimov, V.M.: The principle of convergence "almost everywhere" in Lie groups.

Math. USSR Sbornik 20 (1973), $543-555$

[24] Maksimov, V.M.: Mathematical expectations for probability distributions on compact groups. Math. Z. (1980)

[25] Mourier, E.: Eléments aléatoires dans un espace de Banach. Ann. Inst. Henri Poincaré 13 (1953), 161 - 244

[26] Mourier, E.: L-random elements and L"-random elements in Banach spaces.

Proc. 3rd Berkeley Sympos. on Math. Statistics and Prob. Vol II $(1956), 231-242$

[27] Pakshirajan, R.P.: An analogue of Kolmogorov's three series theorem for abstract random variables. Pac. J. Math. $13(1963), 639-646$

[28] Ressel, P.: Zufallsgrößen in Fréchet- und Banachräumen. Diploma thesis, Münster (1970)

[29] Sugiura, M.: Unitary representations and harmonic analysis: An introduction. Kodansha Ltd. and John Wiley \& Sons (1975)

[30] Trimèche, K.: Probabilités indéfiniment divisibles et théorème de la limite centrales pour une convolution généralisée sur la demi-droite. In: Séminaire d'Analyse Harmonique, Faculté des Sciences de Tunis, Département de Mathématiques (1976) 
[31] Tutubalin, V.N.: On the limiting behavior of compositions of measures in the plane and space of Lobachevsky. Theory of Prob. and Appl. 7 (1962), $189-196$

[32] Uhlmann, W.: Mittelwerte und ihre Schätzfunktionen bei zirkulären zufälligen Variablen.

Metrika $8(1964), 25-47$

[33] Vilenkin, N.J.: Special functions and the theory of group representations.

Translations of mathematical monographs Vol 22. American Math. Society (1968).

[34] Vogt, H.: Zur Definition und Schätzung von Mittelwerten für zufällige Variable auf der Sphäre. Metrika 16 (1970), $206-235$

[35] Woyczyński, W.A.: Geometry and martingales in Banach spaces. Part II: Independent increments. In: Probability on Banach spaces, 267 - 517. Marcel Dekker (1978) 


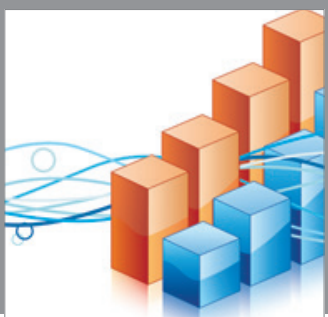

Advances in

Operations Research

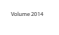

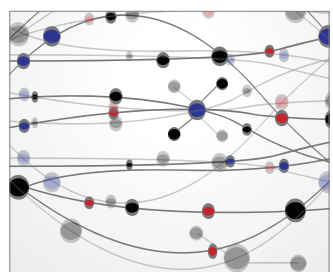

\section{The Scientific} World Journal
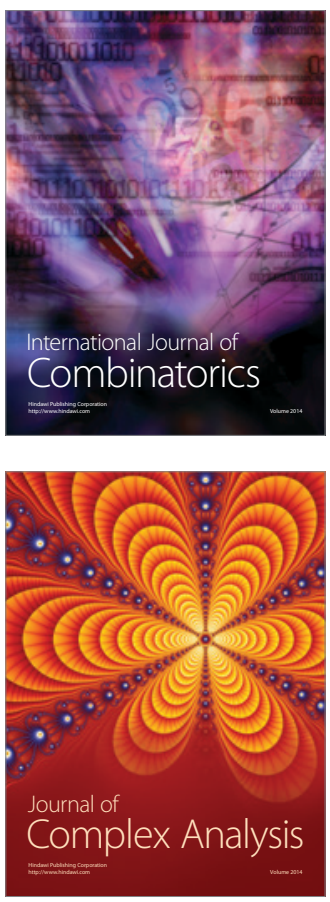

International Journal of

Mathematics and

Mathematical

Sciences
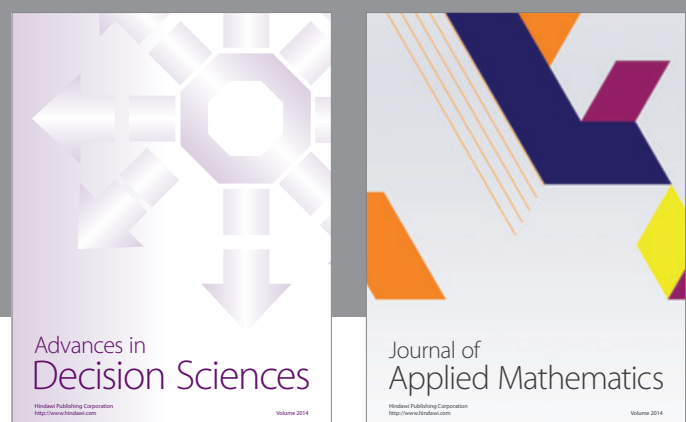

Journal of

Applied Mathematics
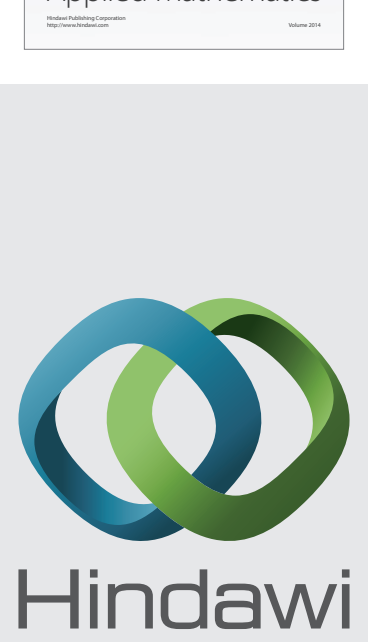

Submit your manuscripts at http://www.hindawi.com
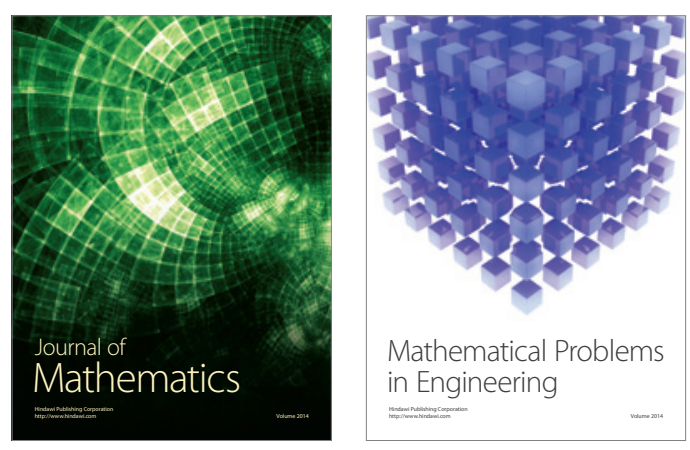

Mathematical Problems in Engineering
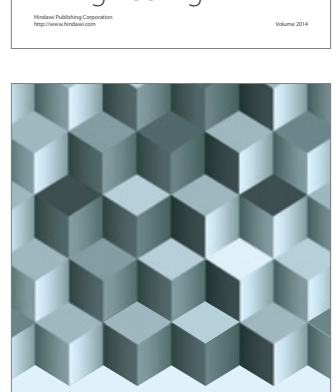

Journal of

Function Spaces
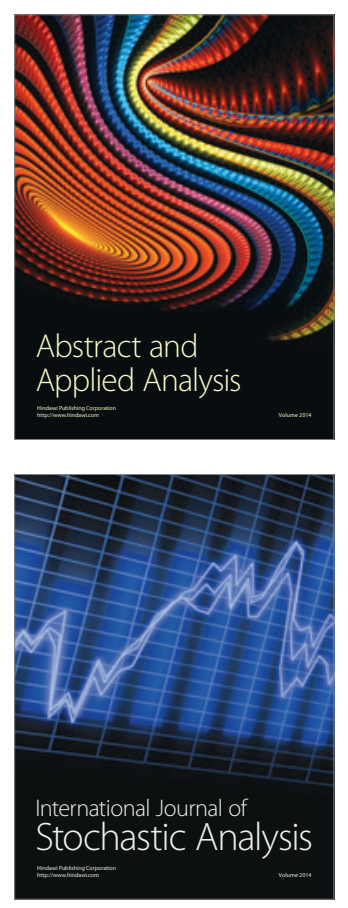

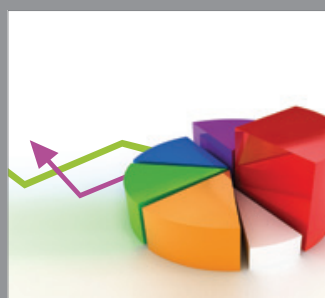

ournal of

Probability and Statistics

Promensencen
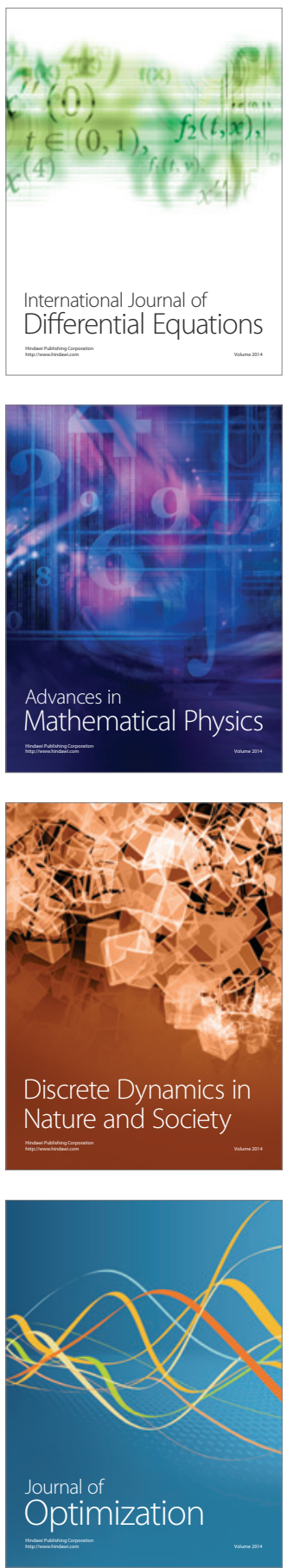